\title{
Frequency dependence of electric field tunability in a photonic liquid crystal fiber based on a gold nanoparticles-doped 6CHBT nematic liquid crystal
}

\author{
Miłosz S. Chychłowski and Tomasz R. Woliński \\ Faculty of Physics, Warsaw University of Technology, Koszykowa 75, 00-662 Warszawa, Poland
}

Received December 07, 2020; accepted December 28, 2020; published December 31, 2020

\begin{abstract}
In this paper, we investigate the external electric field frequency influence on a photonic liquid crystal fiber (PLCF) based on a gold nanoparticles (NPs)-doped nematic liquid crystal (LC) and its response to the external electric field. We used a 6CHBT nematic LC doped with 2-nm gold NPs in a weight concentration of $0.1 \%, 0.2 \%$, $0.3 \%$, and $0.5 \%$.
\end{abstract}

Over the last two decades photonic crystal fibers (PCFs) have gained increasing research interest. Only in the last 10 years more than 17,000 papers have been published on PCFs compared to 11,000 in the first decade [1-3]. The history of liquid crystals (LCs) is much longer and its origins date back to 1888 when the first observations of liquid crystalline phase were made by Friedrich Reinitzer. However, in the last 10 years the number of works published on LC has edged down to 224,000 compared to the previous decade $(229,000)$ [4-6].

Over recent 15 years [7], there has been growing interest in applying LCs into photonics crystal fibers, since manufacturing not only planar waveguides but also optical fibers with LCs combines interesting tuning properties in the form of photonic liquid crystal fibers (PLCFs) [8-9]. In the last 5 years over 1,600 papers have been published on the topic of PCFs and LCs, and in the previous 5 years intervals the number of publications starting with the most recent was as follows: 1,100, 600, 130 , respectively. There is a clear upward trend indicating further development potential in this particular research direction.

Recently our research activities [10-13] have been concentrated on increasing the applicability potential of PLCFs by doping LCs with appropriately selected nanoparticles so that the resulting material as a composite obtains parameters more relevant to its possible application in telecommunications. We have analysed switching times of such systems composed of PCFs and LCs doped with various types of NPs [11-13]. Also, the relationship was investigated between the type, concentration and size of a NP dopand in LC and its influence on the effective PLCF. The results obtained made it possible to significantly reduce the switching times and had a positive effect on electrical control parameters of such systems, allowing to reduce threshold voltages used for their control [11]. Moreover, temperature sensitivity of LCs has been investigated [12] and, in particular, the influence of NPs doping on nematic-isotropic phase transition temperature [12]

In this paper, we present an aspect, not discussed so far, of controlling an external electric field, namely the frequency of an electric field and its influence on the reaction of a system consisting of a PCF composite filled with an NPs-doped LC. The measurement system included a PLCF sample, as well as incoming and outgoing optical fibers. A laser operating at a wavelength of $675 \mathrm{~nm}$ was used as a light source and an Ocean Optics power meter as a detector. The tested sample was placed between two plates to which control voltage was applied after appropriate amplification so that the generated electric field was of the order of $1 \mathrm{~V} / \mu \mathrm{m}$ to obtain reorientation forced by the external electric field (Fig. 1). The range of frequencies used during the experiment was determined by the amplifier high-frequency capabilities and the doped LC relaxation times. The frequency range varied from $10 \mathrm{~Hz}$ to $0.3 \mathrm{MHz}$.

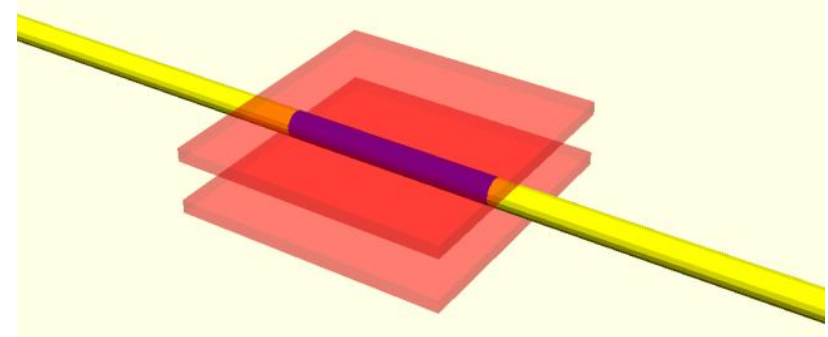

Fig. 1. Setup of PLCF (blue section of yellow optical fiber) with an NPdoped LC response for the electric field with various modulation frequencies placed between two plates (red).

To determine the influence of different frequencies on PLCF responses, a common wavelength in PLCF transmission spectra for all samples is needed. For this purpose, spectral measurements of the transmission of PLCFs filled with a mixture of a 6CHBT nematic LC and 
gold $(\mathrm{Au}) \mathrm{NPs}$ of various concentrations were performed (Fig. 2). As it can be seen in Fig. 2, both transmission spectra without doping and with a $0.5 \%$ doping have similar spectral characteristics. For intermediate values of an Au NPs doped LC, additional transmission bands arise and the existing ones widen relative to the extreme values of an NP Au doped LC. Therefore, the laser source operating at a wavelength of $675 \mathrm{~nm}$ was selected.

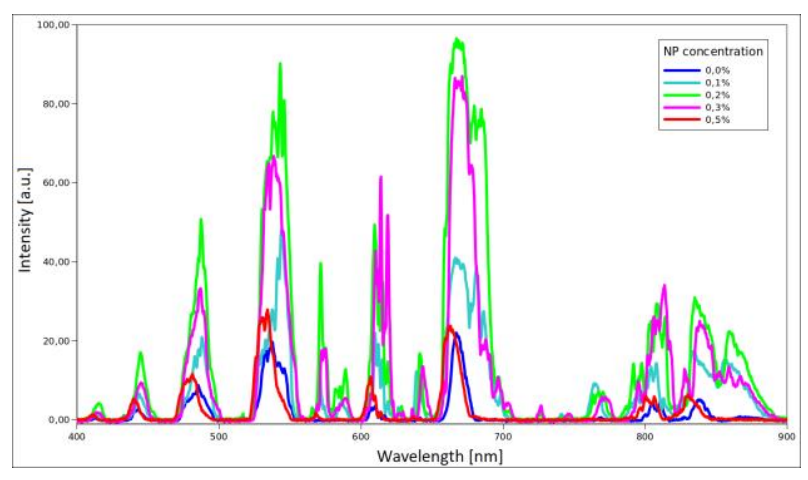

Fig. 2. Transmission spectra for PCF infiltrated with an Au NP doped LC.

Figures 3-5 present the influence of different modulation frequencies of the applied external electric field on response times of the photonics crystal fiber infiltrated with LC mixtures of different AuNP concentrations. The frequency signal in a range from $10 \mathrm{~Hz}$ to $0.3 \mathrm{MHz}$ was generated by a waveform generator and modulated with $1 \mathrm{~Hz}$ for rise and fall time measurements.

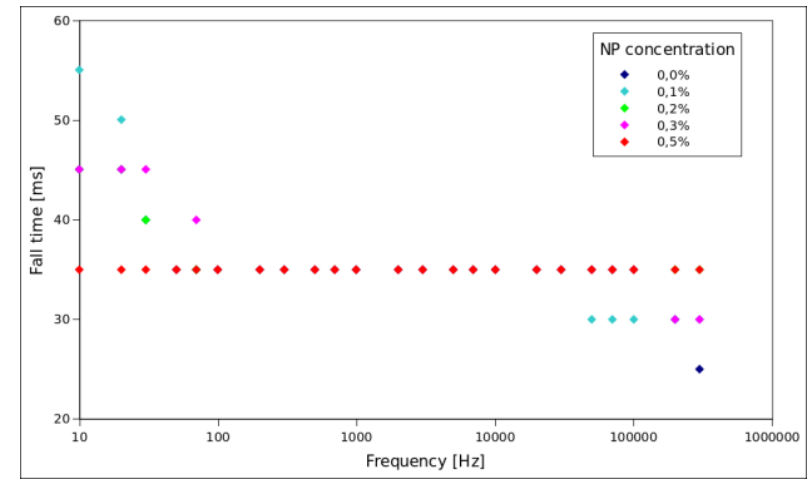

Fig. 3. PLCF fall times induced by an external electric field for a wide frequencies range.

As shown in Fig. 3, for a concentration of $0.5 \%$ wt. $\mathrm{Au}$ NPs in LC, the relaxation times practically do not depend on modulation frequency. However, with a lower percentage of NPs in LC, the relaxation times for higher frequencies decrease from $35 \mathrm{~ms}$ for medium frequencies to $25 \mathrm{~ms}$ for $0.3 \mathrm{MHz}$ for undoped LC. By using lower modulation frequencies of control voltage, there is a clear increase in relaxation times from $35 \mathrm{~ms}$ for medium frequencies to $55 \mathrm{~ms}$ for $10 \mathrm{~Hz}$ for a $0.1 \%$ wt. NP LC doped.

The results of the rise time measurements (Figs. 4 and 5) as a function of the frequency of applied control voltage depend on the percentage of NP in the LC and show a similar relationship as for the relaxation (fall) times. A closer analysis of the results shows that $0.1 \%$ wt. and $0.2 \%$ wt. Au NPs-doped LCs show the shortest rise times. For a doping percentage of $0 \%, 0.1 \%$ wt. and $0.2 \%$ wt. NPs in LCs for lower and medium frequencies, the rise times are relatively constant and are equal to $1.6 \mathrm{~ms}$, $1.1 \mathrm{~ms}, 1.1 \mathrm{~ms}$ respectively, while for higher frequencies there is an increase in rise times to $5.9 \mathrm{~ms}, 4.0 \mathrm{~ms}$ and 4.1 $\mathrm{ms}$ respectively. For a doping percentage of $0.3 \% \mathrm{wt}$. and $0.5 \%$ wt. NPs in LCs, there are large values of rise times for lower frequencies, minimal values for medium frequencies, and a clearly visible increase for higher frequencies. For $0.3 \%$ wt. NP in LC, the rise times are 3 $\mathrm{ms}, 2.2 \mathrm{~ms}$, and $5.7 \mathrm{~ms}$ for low, medium, and high frequencies, respectively. For $0.5 \%$ wt. NP in LC, the rise times are $50 \mathrm{~ms}, 22 \mathrm{~ms}$, and $4.4 \mathrm{~ms}$ for low, medium, and high frequencies, respectively.

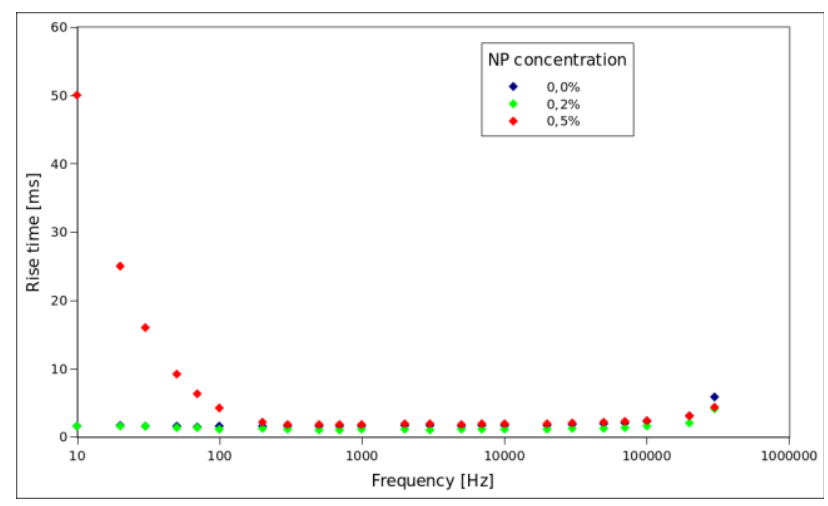

Fig. 4. PLCF rise times induced by an external electric field for a wide frequencies range for concentrations $0.5 \%$ wt. to $0.2 \%$ wt. and $0 \%$ wt.

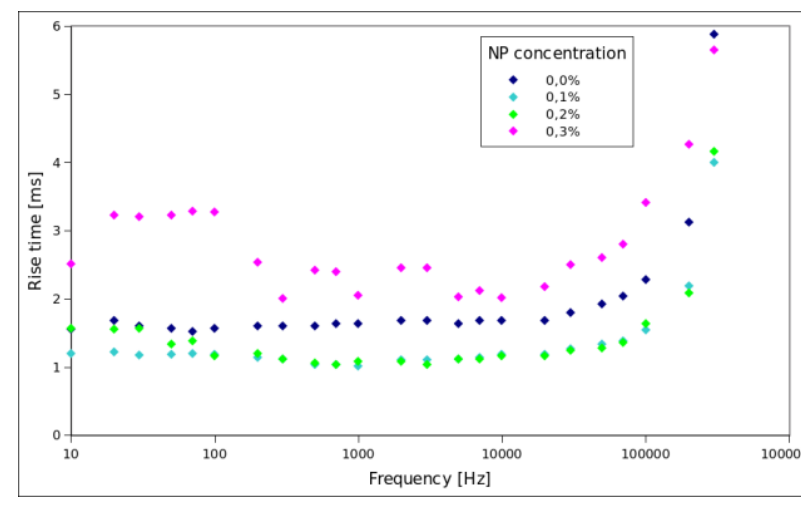

Fig. 5. PLCF rise times induced by an external electric field for a wide frequencies range for concentrations $0 \%$ wt. $-0.3 \%$ wt..

(C) 2020 Photonics Society of Poland 
Based on the results obtained, one can determine a suitable frequency range of control voltage for the PCF infiltrated with an NPs-doped LC and percentage concentration of the dopant in the LC. For the 6CHBT nematic liquid crystal doped with $2 \mathrm{~nm}$ Au nanoparticles, the optimal frequency range for control voltage is between $0.5 \mathrm{kHz}$ and $10 \mathrm{kHz}$. In this range, minimal values of rise and relaxation times can be simultaneously achieved. Due to the characteristics for the rise times, it is possible to determine the optimal percentage of an Au NP dopand in the LC and it is equal to $0.1 \%$ wt. of the Au NP in the 6CHBT LC.

This work was supported by the Grant NCN 2015/19/B/ST7/03650 from the Polish National Science Center.

\section{References}

[1] J. C. Knight, T.A. Birks, P.St.J. Russell, D.M. Atkin, Opt. Lett. 21, 1547 (1996); https://doi.org/10.1364/OL.21.001547

[2] J.C. Knight, T.A. Birks, P.St.J. Russell, J.P. De Sandro, J. Opt. Soc Am. A 15(3), 748 (1998)

[3] S.A. Cerqueira, F. Luan, C.M.B. Cordeiro, A.K. George, J.C. Knight, Opt. Expr. 14(2), 926 (2006).

[4] W. Bragg, Nature 133, 445 (1934); https://doi.org/10.1038/133445a0

[5] J. Kędzierski, K. Garbat, Z. Raszewski, M. Kojdecki, K. Kowiorski, L. Jaroszewicz, W. Piecek, Opto-Electr. Rev. 22(3), 162 (2014).

[6] Y. Li, S.T. Wu, Opt. Expr. 19(9), 8045 (2011).

[7] T. Woliński, S. Ertman, K. Rutkowska, D. Budaszewski, M. SalaTefelska, M. Chychłowski, K. Orzechowski, K. Bednarska, P. Lesiak, Phot. Lett. Poland 11(2), 22 (2019); doi.org/10.4302/plp.v11i2.907.

[8] T.T. Larsen, A. Bjraklev, D.S. Hermann, J. Broeng, Opt. Expr. 11(20), 2589 (2003).

[9] T.R. Woliński, K. Szaniawska, K. Bondarczuk, P. Lesiak, A.W. Domański, R. Dą̧browski, E. Nowinowski-Kruszelnicki, J. Wójcik, Opto-Electr. Rev. 13(2), 59 (2005).

[10] L. Scolari, S. Gauza, H. Xianyu, L. Zhai, L. Eskildsen, T.T. Alkeskjold, S.-T. Wu, A. Bjarklev, Opt. Expr. 17(5), 3754 (2009).

[11] A. Siarkowska, M. Chychłowski, D. Budaszewski, B. Jankiewicz, B. Bartosewicz, T.R. Woliński, Beilstein J. Nanotechn. 8(1), 2790 (2017).

[12] D. Budaszewski, M. Chychłowski, A. Budaszewska, B. Bartosewicz, B. Jankiewicz, T.R. Woliński, Opt. Expr. 27(10), 14260 (2019).

[13] D. Budaszewski, A. Siarkowska, M. Chychłowski, B. Jankiewicz, B. Bartosewicz, R. Dąbrowski, T.R. Woliński, J. Mol. Liq. 267, 271 (2018). 